\title{
BMJ Open University students' understanding and perceptions of schizophrenia in the UK: a qualitative study
}

\author{
Charlotte Cadge, ${ }^{1}$ Charlotte Connor, ${ }^{2}$ Sheila Greenfield ${ }^{3}$
}

To cite: Cadge C, Connor C, Greenfield S. University students' understanding and perceptions of schizophrenia in the UK: a qualitative study. BMJ Open 2019;9:e025813. doi:10.1136/ bmjopen-2018-025813

- Prepublication history and additional material for this paper are available online. To view these files, please visit the journal online (http://dx.doi org/10.1136/bmjopen-2018025813).

Received 6 August 2018 Revised 21 November 2018 Accepted 21 January 2019

Check for updates

(C) Author(s) (or their employer(s)) 2019. Re-use permitted under CC BY-NC. No commercial re-use. See rights and permissions. Published by BMJ.

${ }^{1}$ College of Medical and Dental Sciences, University of Birmingham, Birmingham, UK ${ }^{2}$ Mental Health and Wellbeing, University of Warwick Warwick Medical School, Coventry, UK ${ }^{3}$ Institute of Applied Health Research, University of Birmingham, Birmingham, West Midlands, UK

Correspondence to

Dr Sheila Greenfield;

s.m.greenfield@bham.ac.uk

\section{ABSTRACT}

Objective To explore lay understanding and perceptions of schizophrenia in university students.

Design Qualitative study using semi-structured interviews and thematic analysis.

Setting The University of Birmingham, West Midlands. Participants 20 UK home students of white British $(n=5)$, Indian $(\mathrm{n}=5)$, Pakistani $(\mathrm{n}=5)$, African Caribbean $(\mathrm{n}=4)$ and dual white British and African Caribbean ethnicity $(n=1)$. Results Findings revealed a lack of knowledge about schizophrenia, particularly the negative symptoms that were not mentioned. There were mixed ideas on the causes and sources of available help for schizophrenia; however, positively many said they would consult their general practitioner. While there was a general misconception among the students that schizophrenia caused multiple personalities and was a dangerous illness, there were some differences in perceptions and understanding between ethnic groups, with more Indian students perceiving upbringing as a causal factor in the development of the illness and more Pakistani students perceiving possession by a spirit as a cause.

Conclusions The university students interviewed lacked knowledge about schizophrenia and stigma was widespread, both of which may delay help-seeking. Public health campaigns educating young people about schizophrenia are required to improve early identification and intervention and improve outcomes. Further research exploring ways to effectively tackle stigma is also required.

\section{INTRODUCTION}

Schizophrenia is a mental health condition which significantly alters a person's perception, thoughts, mood and behaviour. 'There are a variety of 'positive symptoms' where a change in behaviour or thought occurs, such as hallucinations and delusions, as well as 'negative symptoms' where there is a lack of or withdrawal of a function expected in a healthy individual, such as emotional apathy and social withdrawal. ${ }^{1}$

While there is currently no cure for schizophrenia, early diagnosis and management are key to improving long-term outcomes. ${ }^{2-4}$ However, many are diagnosed and therefore treated over a year after the onset of psychotic symptoms. ${ }^{4}$ This is due to, at least in part,

\section{Strengths and limitations of this study}

- This study provides the first in-depth qualitative exploration of UK public perceptions of schizophrenia since 1999 and is the first qualitative exploration of university students' perceptions of schizophrenia within the UK allowing insight into the views of a highly educated group.

- This study used robust methodology with analyst triangulation, member validation and a reflexive approach increasing rigour and validity of findings.

- While ethnic minorities are often under-represented in healthcare research, purposive sampling of participants ensured comparable numbers of ethnicities were interviewed.

- All participants were recruited from a single university and were thus all highly educated which may limit transferability.

- Snowball sampling and advertising the study as looking at mental illnesses could both lead to potential sampling bias.

poor help-seeking in those experiencing the symptoms of schizophrenia, ${ }^{5}$ which may be attributed to poor mental health literacy or fear of the stigma attached to the disease. Delayed help-seeking increases the duration of untreated psychosis (DUP), leading to an increased risk of suicide, lower levels of recovery and poorer treatment outcomes. ${ }^{346}$

Mental health literacy refers to 'knowledge and beliefs about mental disorders which aid their recognition, management or prevention'. ${ }^{7}$ This includes the ability to recognise disorders, obtain relevant information and seek appropriate help. Insight into lay mental health literacy for schizophrenia is therefore important in identifying potential barriers to early consultation and diagnosis. Public health campaigns and education aiming to combat these potential barriers may help to improve earlier consultation and therefore reduce DUP. Our recent investigation of poor help-seeking and long DUP resulted in Birmingham and Solihull Mental Health Foundation Trust implementing a Psychosis 
Awareness Public Health campaign alongside the introduction of a direct referral service. ${ }^{8}$

Symptoms of schizophrenia typically emerge in late adolescence and early adulthood, ${ }^{1}$ making early intervention most relevant at this age. Investigating mental health literacy in young adults is thus important to determine whether they would recognise symptoms in themselves, and importantly their peers, as those with schizophrenia may lack insight. ${ }^{9}$

Despite schizophrenia affecting $1 \%$ of the population, ${ }^{10}$ research has shown low levels of general public understanding and common misconceptions. ${ }^{11}$ While one study focusing on UK university students found $82.4 \%$ of respondents had heard of schizophrenia, it was unclear if they would be able to define it or had correct knowledge. ${ }^{12}$ The majority of previous research has been conducted outside the UK, where different education and mental health campaigns may result in considerable differences in understanding. There is a lack of research focusing on students despite this group's increased vulnerability to mental health difficulties. ${ }^{13}$ Further, the limited research focusing on the UK has predominantly used quantitative methods, measuring responses to a number of preformed statements on a questionnaire. ${ }^{14-16}$ There appears to be no qualitative research into UK university students' understanding or perceptions of schizophrenia. While useful, quantitative research does not explore unprompted knowledge, which may be more relevant when exploring mental health literacy and help-seeking behaviours. The most recent in-depth qualitative exploration of UK public perceptions of schizophrenia was published in $1999^{17}$ and focused specifically on the impact of religion on beliefs towards causes and treatment. Significant changes in understanding can occur over time with evolving education and new campaigns. ${ }^{18}$ There is therefore a need for up-to-date qualitative exploration, as emphasised in more recent research. ${ }^{19}$

Research focusing on students to explore gaps in the knowledge of this group and identify commonly held misconceptions can help inform targeted public campaigns to improve early diagnosis and treatment of schizophrenia, resulting in improved outcomes. Therefore, this study explores the understanding and perceptions of schizophrenia in UK university students.

\section{AIMS}

The primary aims of the study were

- To explore lay perceptions of the term schizophrenia in UK university students.

- To explore university students' understanding of the symptoms and treatment of schizophrenia and who to consult.

The secondary aim of the study was to explore the impact of ethnicity on perceptions and understanding of schizophrenia.

\section{METHODS}

\section{Participants}

Participants were recruited from a convenience sample ${ }^{20}$ of students at the University of Birmingham. International students were excluded in order to focus on UK perceptions. In order to focus on lay perceptions, students with a close friend or family member with schizophrenia or those studying psychology or a course in the College of Medicine and Dentistry were also excluded. White British, Indian, Pakistani and African Caribbean students were recruited, as the four most prevalent ethnicities in the UK. ${ }^{21}$ This was to allow for exploration of differences between ethnicities but is also representative of the West Midlands, which is the second most ethnically diverse area in the UK after London. ${ }^{21}$

\section{Recruitment}

The study was advertised through flyers, which were distributed by hand on the university campus, and also through posts on a number of University of Birmingham Facebook groups. ${ }^{22}$ The study was advertised as looking at mental illnesses in general to ensure participants did not research schizophrenia prior to the interview. Interested students were emailed an information sheet and eligibility questionnaire, which included questions on the student's gender, age, religion, course studied, ethnicity as based on census classification, ${ }^{21}$ and information on whether a close friend or family member of theirs has had a mental illness. This ensured participants fulfilled the eligibility criteria and allowed purposive sampling ${ }^{20}$ based on ethnicity and gender. Where multiple students with the desired characteristics were interested, the first to return the eligibility questionnaire was selected. Due to difficulties recruiting sufficient numbers of each ethnicity, snowball sampling ${ }^{20}$ was also employed to recruit one student each of Indian and Afro-Caribbean ethnicity, with participants asked if they knew of students with the desired characteristics to take part. Twenty students were interviewed, which is in line with sample size recommendations for a study of this type to allow for data saturation to occur. ${ }^{23}$

\section{Data collection}

Face-to-face, semi-structured interviews were used due to the sensitive nature of discussing perceptions of mental illnesses and to distinguish individuals' baseline knowledge. Interviews took place in a private room at the University of Birmingham Medical School throughout February and March 2017 with no repeat interviews undertaken. All interviews were conducted by CCa, a white British female medical student intercalating in Public Health and Population Sciences. Interviews ranged between 15 and $46 \mathrm{~min}$ with a mean average of $25 \mathrm{~min}$. The researcher knew none of the participants prior to study initiation. A topic guide (online supplementary file 1), generated using relevant literature, ${ }^{717}$ was used to ensure consistency across interviews and ensure key areas were covered to meet the study objectives. The topic guide was piloted in university students known to the researcher to ensure 
the ease of understanding. Data from pilot interviews was not included in analysis. Written informed consent was obtained prior to starting each interview. Following each interview, participants received a $£ 15$ Amazon voucher in appreciation of their time and the interviewer took field notes to record emerging ideas and aid later interpretation of transcripts.

\section{Data analysis}

Data was thematically analysed with codes and themes identified inductively, on the basis of the data. ${ }^{24}$ Interviews were audio-recorded and transcribed verbatim by the researcher, with audio recordings listened back to in order to ensure transcripts were correct. Field notes were then reviewed and transcripts read twice for familiarisation. ${ }^{24}$ Following familiarisation open coding was done manually, with codes written in the printed transcript margin. Codes were further refined as the analysis progressed and sorted into categories and themes with the aid of mind maps and a Microsoft Excel spreadsheet. No new codes or categories emerged in the final two transcripts, confirming data saturation was met across the data set. ${ }^{25}$ Codes were entered into a Microsoft Excel spreadsheet per participant to allow deviant cases to be identified and allow comparisons to be made between participants and ethnicities.

To ensure quality and rigour of the analysis process, analyst triangulation occurred. ${ }^{26}$ Two transcripts were independently coded by SG, an experienced qualitative researcher, who met with CCa to discuss and agree on analysis and identified codes. The researchers further met to discuss interpretation of codes and subsequent refinement of themes to ensure these were appropriately and clearly defined. Additionally, each participant was emailed a summary of the main themes and points emerging from their individual interviews for member validation. ${ }^{25}$ Nineteen participants responded $(95 \%)$, all stating they were a correct interpretation of their viewpoints, further substantiating data analysis. ${ }^{25}$ Throughout data collection and analysis, the researcher worked reflexively, being particularly sensitive to the potential impact on participants of talking to someone from a different cultural background.

\section{Patient and public involvement}

The public were not involved in the development of the research question, study design or recruitment of participants. Participants were emailed a summary of the themes from their interview and an initial manuscript at a later date.

\section{RESULTS}

Pseudonyms are used in the place of participant names alongside quotations to maintain confidentiality. The 20 students interviewed ranged in age from 18 to 22. Five students of white British, Indian and Pakistani ethnicity were interviewed. Due to difficulties in recruitment, only

\begin{tabular}{|c|c|c|}
\hline Ethnicity & $\begin{array}{l}\text { Women : } \\
\text { men split }\end{array}$ & Religion represented \\
\hline White British & $3: 2$ & $\begin{array}{l}\text { Christianity (2), Judaism (1), } \\
\text { Atheism (2) }\end{array}$ \\
\hline Indian & $2: 3$ & Islam (1), Sikhism (3), Hinduism (1) \\
\hline Pakistani & $3: 2$ & Islam (5) \\
\hline African Caribbean & $3: 1$ & Christianity (3) and Islam (1) \\
\hline $\begin{array}{l}\text { White British and } \\
\text { African Caribbean }\end{array}$ & $0: 1$ & Christianity (1) \\
\hline
\end{tabular}

four African Caribbean students were recruited and one participant of dual white British and African Caribbean ethnicity. Six religious groups were represented with Christian, Jewish, Muslim, Sikh, Hindu and atheist participants. Information about the gender and religious views of participants within each ethnicity is given in table 1 .

Five themes, each with subcategories, emerged from the data: lack of knowledge; causes of schizophrenia; help; effects of schizophrenia; stigma. Figure 1 summarises the themes with their corresponding sub-categories and the impact ethnicity had on these.

\section{LACK OF KNOWLEDGE}

Throughout the interviews, there was a general lack of knowledge when discussing all areas of schizophrenia, with misconceptions being common.

\section{Unawareness}

The majority of students had very little prior knowledge of schizophrenia with one student confusing it with Parkinson's disease.

Isn't it the illness where your hands shake uncontrollably? (Arjun, Indian)

Further, students lacked confidence in their perceptions about the illness and were often reluctant to answer.

If I'm wrong I wouldn't be surprised. (Jack, white British)

\section{Misconceptions}

Nine students believed schizophrenia caused people to have 'split' or multiple personalities.

In terms of schizophrenia as a term, I really just understand it to be someone with like split personalities. (Keshini, Indian)

The perception of schizophrenia as something that 'doesn't exist' was mentioned by seven students. This was partly attributed to its intangibility.

Cause people think it's all in your head ... maybe it doesn't exist and you just need to get over it. (Emily, white British) 


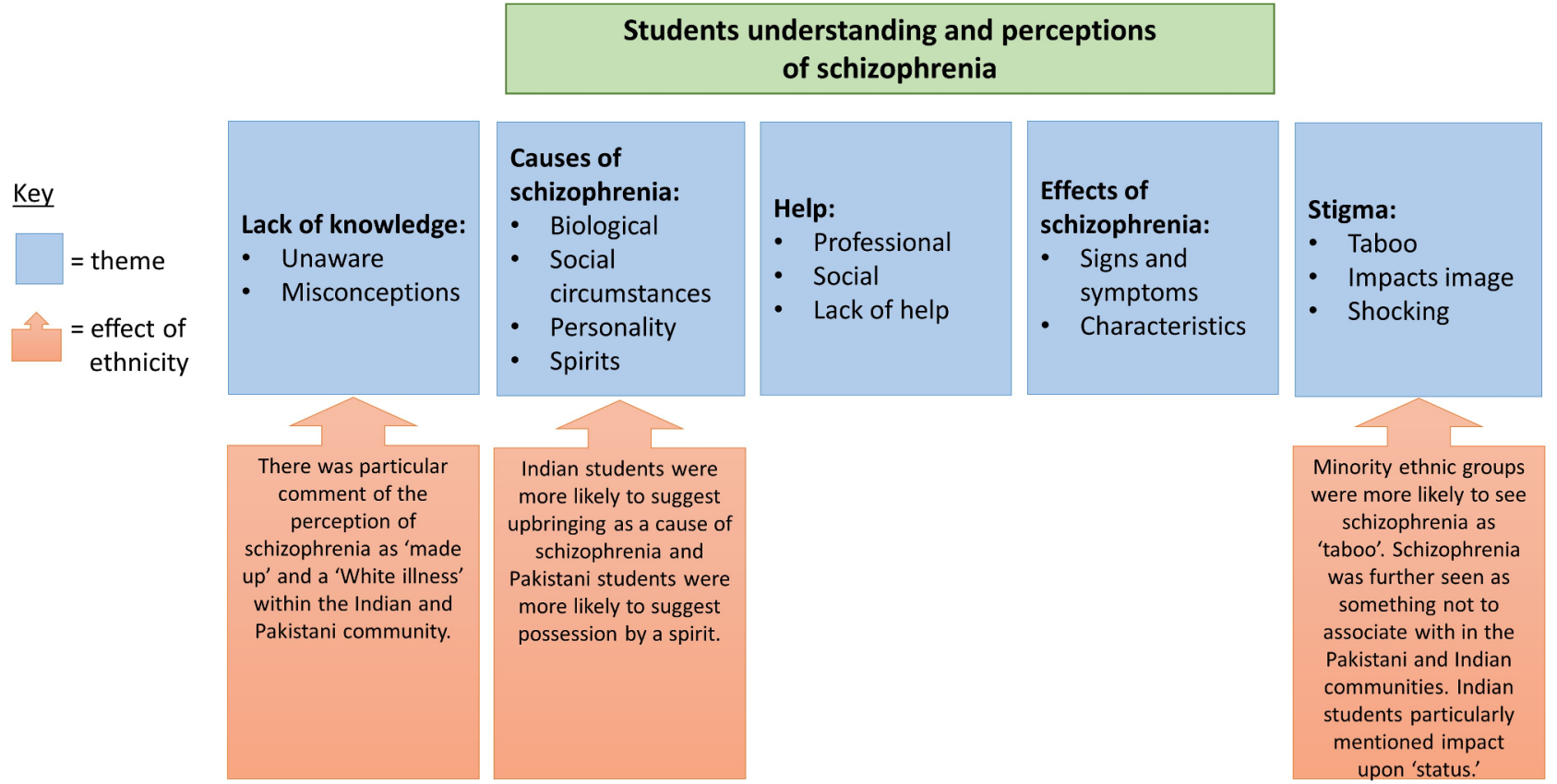

Figure 1 Summary of themes and impact of ethnicity on these. A diagram summarising the themes that emerged from the data and impact ethnicity had on these. Each blue box represents one of the five main themes with its corresponding sub-categories. Each orange block arrow shows the impact ethnicity had on the reciprocal theme. Ethnicity did not impact perceptions of available help or effects of schizophrenia.

Both Indian and Pakistani students mentioned the perception of schizophrenia as 'made up' within the wider Indian and Pakistani community, which they partly put down to a lack of education of and exposure to mental illnesses in older generations.

In my community... they're like this doesn't exist ... it's a white person thing. (Waqas, Pakistani)

\section{CAUSES OF SCHIZOPHRENIA}

The majority of students were unaware of the potential causes of schizophrenia when first asked. Beliefs about causes, with further prompting, are presented in table 2 along with numbers of students holding the belief, to demonstrate typicality of views. ${ }^{27}$

Most students believed social circumstances or a biological component could cause schizophrenia and eight students believed personality characteristics could contribute, including being introverted or aggressive. Of the four students who believed upbringing could contribute, three were Indian.

While there were students of each ethnicity believing schizophrenia could have a spiritual cause, these were mostly of Pakistani heritage believing mental illnesses could be caused by possession by a 'jinn' (spirits found in Islamic and Arabic writings that are able to appear in human or animal form and possess humans). ${ }^{28} \mathrm{~A}$ number of these students further commented on how, while as a Muslim they believed mental illnesses could be caused by possession by a jinn, they also thought that mental illnesses are often confused with possession.
There's a lot of confusion in our culture... I do believe that there are certain times when mental illnesses are seen to be possessions when they're actually not. (Ibrahim, Pakistani)

Comment was also made on how 'blaming' possessions can be popular within the Indian community. This was believed to remove blame from the individual or their family through attributing an external cause and allowing a 'quick fix' through a ritual to remove the spirit.

I know in the Asian community if someone has mental illness they'll be like oh it was something paranormal... they really push it as something external because I think it's that fear that it is characteristic of you. (Anshula, Indian)

\section{SOURCES OF HELP}

On the whole, students were unaware of what help was available for schizophrenia. The need for expert help was emphasised by 17 students and the importance of social support was emphasised by 12 students. Many comments were made about difficulties in treating schizophrenia including issues with the current system within the National Health Service.

\section{Professional}

Sixteen students said they would consult their general practitioner (GP); however, a number of students said this was only due to a lack of alternative options. Many participants, especially of white British ethnicity, commented on 
Table 2 Perceived causes of schizophrenia

\begin{tabular}{|c|c|c|c|}
\hline Cause of schizophrenia & Subcategory & Frequency stated & Supporting quotations \\
\hline \multirow[t]{4}{*}{ Biological } & & 19 & \\
\hline & Genetic & 16 & $\begin{array}{l}\text { 'I can't help but think there might be a genetic component to } \\
\text { it.' (Harry, white British) }\end{array}$ \\
\hline & Neurological & 7 & $\begin{array}{l}\text { 'I think there's like a biological cause ... like maybe to do witl } \\
\text { your brain functioning.' (Serena, African Caribbean) }\end{array}$ \\
\hline & Drugs & 2 & $\begin{array}{l}\text { 'I think schizophrenia is something which you can cause to } \\
\text { yourself, perhaps through drug abuse.' (Ibrahim, Pakistani) }\end{array}$ \\
\hline
\end{tabular}

$\begin{array}{lrrr}\text { Social circumstances } & 16 & & \\ & \text { Traumatic event } & 9 & \text { 'I guess if someone's been through something quite traumatic }\end{array}$
... then it could trigger it.' (Noor, Pakistani)

$\begin{array}{ll}\text { Isolated } & 7 \\ \text { Upbringing } & 4\end{array}$

'People who often just find themselves alone ... that could lead to schizophrenia.' (Tia, African Caribbean)

'It depends what kind of environment you've been brought up in... I do know children who are kind of brought up in more kind of restricted environments that tends to bring about mental illness.' (Anshula, Indian)

\begin{tabular}{|c|c|c|c|}
\hline & Social class & 4 & $\begin{array}{l}\text { 'If you're raised from a hard environment, working class say } \\
\text { and you're living a hard life just about getting by... that type of } \\
\text { stuff could lead to it.' (Waqas, Pakistani) }\end{array}$ \\
\hline & Pressure & 3 & $\begin{array}{l}\text { 'I think high pressure could contribute to it.' (Jack, white } \\
\text { British) }\end{array}$ \\
\hline \multirow[t]{4}{*}{ Personality } & & 8 & \\
\hline & Introvert & 4 & $\begin{array}{l}\text { 'If you have the personality of someone who's very alone } \\
\text {... keeping alone and secluded I feel that could lead to } \\
\text { schizophrenia.' (Zahid, Pakistani) }\end{array}$ \\
\hline & Aggressive & 3 & $\begin{array}{l}\text { 'If someone's more towards the aggressive side, the angry } \\
\text { side and stuff I think that contributes.' } \\
\text { (Michael, white British and African Caribbean) }\end{array}$ \\
\hline & Risk taker & 1 & $\begin{array}{l}\text { 'If their personality involves being someone who takes risks... } \\
\text { that would make them more likely to have it.' (Ibrahim, } \\
\text { Pakistani) }\end{array}$ \\
\hline
\end{tabular}

issues with the current healthcare system including GPs' lack of expertise.

They don't help ... they're kind of just the dispensers of the forms ... they're not the person who can actually help ... they're not specialized in mental health they're specialized in oh you have a cold. (Grace, white British)

Five students said they would consult university services for help and 11 said they would research online for information about schizophrenia and sources of help.

On the internet you can find a lot of the NHS services. (Ibrahim, Pakistani)

All students perceived medication as helping in some way with comments made on how it can be reliable as a 'proven' treatment. Five students, however, also commented on issues associated with medication including negative effects and dependence.
I think medication kind of masks the issue rather than kind of trying to resolve where it started from... I think it's very easy to get hooked. (Keshini, Indian)

Six students commented on the ineffectiveness of both medication and GPs, as being better suited to physical illnesses. Twelve students perceived therapy with psychologists or psychiatrists as the best help available due to their expertise.

With something that's a mental illness there has to be some therapy from like experts. (Waqas, Pakistani)

\section{SOCIAL}

Social support was perceived as key in helping someone with schizophrenia to recover or cope. Many students of each ethnicity believed religion could aid recovery through prayer, as well as providing hope and a wider 
support network. Moreover, four students viewed social support as more important than professional help.

I feel like medical intervention ... can't compare to the support of friends and family, I feel like that could be a more important medicine. (Eve, African Carribean)

The importance of personal internal coping mechanisms was also discussed, with nine students, particularly four of Indian ethnicity, further discussing a desire not to consult due to the belief they could get over issues alone.

It's all well saying I'd probably seek help but I honestly don't think I would ... you think you're strong enough to probably get over it yourself. (Keshini, Indian)

\section{Lack of help}

Many students commented on the lack of help available, perceiving schizophrenia as 'always there' and difficult to treat, particularly in comparison to physical illnesses.

I'm not sure if it ... goes away like a cold that you have for 2 weeks, I think it's something that you sort of always live with. (Eve, African Caribbean)

\section{EFFECTS OF SCHIZOPHRENIA}

Effects of schizophrenia were discussed including the expected signs and symptoms and characteristics.

\section{Signs and symptoms}

Perceived signs and symptoms are presented in table 3. On the whole students were unaware what specific symptoms were associated with schizophrenia with nine commenting on the lack of visible symptoms. Of the 20 students, 11 were aware of psychotic symptoms of either hallucinations, delusions or both. Many students associated schizophrenia with abnormal behaviour and mood swings, often alongside the idea of multiple personalities.

\section{Characteristics}

Those with schizophrenia were often viewed as dangerous, vulnerable and 'out of touch'.

\section{Dangerous}

Thirteen students viewed those with schizophrenia as dangerous, associating the disease with 'violence' and 'rage'. Four students additionally commented on how those with schizophrenia can harm and impact on others.

\section{Table 3 Perceived signs and symptoms of schizophrenia}

\begin{tabular}{|c|c|c|}
\hline Signs and symptoms & Frequency stated & Supporting quotations \\
\hline Hallucinations, including voices & 9 & $\begin{array}{l}\text { 'I know it can cause you to have hallucinations so you see people, you } \\
\text { might hear people who aren't actually there.' (Grace, white British) } \\
\text { 'I think it's when people have voices in their head.' (Zahid, Pakistani) } \\
\text { 'I know in some cases people hear voices.' (Tia, African Caribbean) } \\
\text { 'I can think of symptoms like one could be hearing voices or } \\
\text { hallucinations.' (Noor, Pakistani) }\end{array}$ \\
\hline Not visible & 9 & $\begin{array}{l}\text { 'You don't know I might be having thoughts in my head like you can't see } \\
\text { that.' (Grace, white British) } \\
\text { 'It's invisible in the sense that like just seeing the person you can't really } \\
\text { tell.' (Eve, African Caribbean) }\end{array}$ \\
\hline Paranoia & 4 & $\begin{array}{l}\text { 'Schizophrenia has a big connection to paranoia in my mind.' (Ibrahim, } \\
\text { Pakistani) }\end{array}$ \\
\hline Mood swings & 4 & $\begin{array}{l}\text { 'I'd expect like constant mood swings and like behaviour that you } \\
\text { wouldn't normally expect of that person.' (Michael, white British and } \\
\text { African Caribbean) } \\
\text { 'At times be very calm, stoic, genuinely kind of happy almost and then } \\
\text { other times for like the smallest stimuli be really erratic or angry or almost } \\
\text { violent.' (Toson, African Caribbean) }\end{array}$ \\
\hline Talk to self & 3 & $\begin{array}{l}\text { 'I'd expect erratic behaviour and maybe talking to themselves.' (Tia, } \\
\text { African Caribbean) }\end{array}$ \\
\hline
\end{tabular}


I think schizophrenia if you describe it as a colour it's like red so it's just all about rage and stuff ... and you know someone gets enraged they normally take it out on other people. (Waqas, Pakistani)

The perception was furthered by the common view of those with schizophrenia as erratic and not in control.

I would say [schizophrenia] is a stronger version of someone ... who's not really in control of their actions. (Raj, Indian)

\section{Vulnerable}

Nine students perceived those with schizophrenia as vulnerable, seeing schizophrenia as a challenging and frightening disease to go through. Moreover, seven students associated schizophrenia with isolation, perceiving those with schizophrenia as lonely and lacking support.

I'd expect them to be like quite isolated and just troubled. (Noor, Pakistani)

A number of students also viewed those with schizophrenia as insecure, with three students seeing an individual's insecurities and fears as contributing towards causing the condition. Two students partly attributed individuals' doubts about themselves to the stigma surrounding the disease and others' negative perceptions.

I'd expect them to be quite nervous and not too sure of themselves. (Jack, white British)

\section{Out of touch}

There was also a view of those with schizophrenia as being 'out of touch' with the perception schizophrenia caused you to 'lose yourself'.

As in they're not really themselves... it's not really them, that's how I look at it. (Raj, Indian)

Seven students thought individuals would have a lack of awareness, particularly a lack of insight into their condition.

I think it can be a very confusing mental illness to go through, almost gives the impression there is no mental illness that it's just you. (Harry, white British)

\section{STIGMA}

The majority of students saw schizophrenia as a very stigmatising condition with many negative connotations.

\section{Taboo}

Many students perceived schizophrenia as something that fails to be discussed in the community. This was partly attributed to the personal and private nature of the disease but also how mental illnesses, particularly schizophrenia, are often considered a 'taboo' topic.
In my friendship group ... you don't really talk about mental health ... it's almost like a taboo. (Grace, white British)

While students from all ethnicities saw schizophrenia as a taboo topic, particular comments were made regarding the fact it was not discussed within the Indian, Pakistani and African Caribbean communities.

I feel like mental illness isn't really discussed much in like the black community. (Serena, African Caribbean)

Moreover, a number of Pakistani and Indian students commented on the perception of schizophrenia as something not to associate with, partly down to its impact on image and also the perception of individuals as dangerous.

The way I've heard about it is very much it's a bad thing, don't talk to so and so. (Anshula, Indian)

\section{Impacts on image}

Many students saw schizophrenia as having an impact on image or 'status' with six students perceiving it as a weakness. A number of students said they would be reluctant to tell anyone due to a fear of being judged.

I think it's still considered like a badge of shame compared to other mental illnesses. (Harry, white British)

The impact on 'status' was particularly mentioned by Indian students, with three students seeing it as a reason to hide the disease. This was in part down to the perception of those with mental illnesses as unfit to be parents, and the idea that upbringing and parents can be to 'blame'.

Marriage is such a kind of fundamental part of our culture, if someone isn't kind of mentally healthy it kind of ruins their chances of getting married. (Anshula, Indian)

\section{Shocking}

Six students saw schizophrenia as something that shocks people with one student commenting on the fact they were taken aback when told the interview would be about schizophrenia. This was partly attributed to the perception of schizophrenia as scary, but also misunderstood, with people scared of what they don't understand.

Even saying the word schizophrenia sort of takes you back every time you say it. (Keshini, Indian)

\section{DISCUSSION}

\section{Main findings}

This study highlighted a lack of knowledge and misconceptions about schizophrenia among the university students interviewed, a highly educated group of the population. While many students were aware of schizophrenia's association with psychotic symptoms, there was no mention of 
negative symptoms associated with the condition, which is consistent with previous research. ${ }^{12}$ Awareness of negative symptoms, such as social withdrawal or sleep disturbances, ${ }^{1}{ }^{2930}$ is particularly important as these symptoms can often be mistaken as usual young adult behaviour and delay help-seeking. Moreover, negative symptoms are often the first to occur in schizophrenia and can precede psychotic symptoms by a number of years, ${ }^{29} 30$ making their recognition particularly important for early intervention. Misconceptions about schizophrenia were common, with students associating it more with multiple personalities than any single symptom. This supports previous UK research, which found that multiple personalities was the most frequent association of schizophrenia. ${ }^{31}$ This common belief, alongside a lack of knowledge, may further contribute to delayed help-seeking due to confusion over the symptoms of schizophrenia and an inability to recognise its onset. Furthermore, associating schizophrenia with multiple personalities may contribute to ideas of unpredictability and danger, which have previously been found as the most prevalent negative associations of schizophrenia ${ }^{11}$ and were frequently mentioned in this study.

Most students perceived schizophrenia as stigmatising with perceptions of the disease as shocking and a weakness, impacting on image and status. Further, even in this highly educated cohort most students perceived schizophrenia as dangerous, with associations of violence and unpredictability, despite the fact most with schizophrenia are not violent or dangerous. ${ }^{32}$

It was promising that the majority of students said they would consult their GP and correctly viewed schizophrenia as a serious disease requiring expert help. This goes against previous research, which showed many students were unaware of where to turn for help with mental illnesses and would prefer to talk to family and friends than medical professionals including GPs. ${ }^{33}$ Potential barriers to help-seeking included worries over, and negative perceptions of, GPs and medication. These worries were common and are consistent with our previous qualitative work with first-episode psychosis ${ }^{34}$ where participants felt their GP would simply refer them on or prescribe drugs due to a lack of expertise. This perception may be further compounded by the common view that schizophrenia is especially difficult to treat and 'always there' despite evidence showing a significant proportion of treated cases achieve favourable outcomes and even full recovery. ${ }^{35}$ The belief schizophrenia does not exist or is 'all in your head' is a particularly important perception that emerged from this study which may further delay medical consultation, alongside the belief individuals can 'get over it alone'.

The students we interviewed included participants from four different ethnic groups. Confusion between mental illnesses and possession by spirits and stigma were things that were of relevance to the Indian and Pakistani students we interviewed. Stigma within the community was particularly relevant to those of Indian heritage who talked about how they felt the illness could impact on marriage prospects. Family upbringing was also seen by this group as playing a significant role in the development of schizophrenia, which may reveal an extension of courtesy stigma $^{36}$ to the wider family. This is in line with previous research, which has found stigma to be so extreme within Asian communities that it impacts on the entire family's marriage prospects. ${ }^{37}$ In this study, a number of Indian and Pakistani students further commented on the perception of schizophrenia as something not to associate with. Societal stigma can be a particular barrier to early consultation due to those with schizophrenia concealing symptoms or failing to seek help due to embarrassment or shame. ${ }^{37}$ Further, it may prevent individuals seeking support from their family and peers. While previous research has found profound community stigma within the African Caribbean community ${ }^{17}$ there was less comment on this within this study. Comment was made, however, on the perception of schizophrenia as a more 'taboo' topic within each of the minority ethnic groups, which may further prevent people seeking support.

Contrary to previous research, ethnic background did not have an impact on awareness of signs and symptoms ${ }^{19}$ or available help. ${ }^{17}$ This may be partly down to the higher educational attainment of participants, or differences in the way this was explored in previous studies, which mostly analysed preformed responses to vignettes. ${ }^{19}{ }^{38}$ It is important to note that while differences were found between ethnicities variation was also apparent within ethnic groups. For example while a number of Pakistani students believed jinns could cause schizophrenia, not all did. Therefore, while differences in perceptions should be taken into consideration, it is essential not to generalise or stereotype.

\section{Strengths and limitations}

To the best of our knowledge, this study is the first in-depth exploration of perceptions of schizophrenia within the UK since 1999 and the first qualitative study of UK university students. This is a key strength of the research as interviews allow exploration of knowledge and provided further insight into perceptions of schizophrenia and the complexities surrounding its stigma, including the perception of schizophrenia as 'made up'. Further strengths include the use of member validation, cross-checking of analysis by an experienced qualitative researcher and the fact data saturation of themes was met. Our success in recruiting similar numbers of each ethnicity is an additional strength; minority ethnic groups are often under-represented in healthcare research. ${ }^{39}$

However, there are also a number of limitations. Participants were recruited from one setting and all in higher education. While qualitative research does not aim to be generalisable, ${ }^{26}$ education and age have been shown to impact on mental health literacy. ${ }^{11419}$ This suggests there could be lower levels of knowledge about schizophrenia in the wider population. Additionally, the study was advertised as looking at mental illnesses, which could result in 
the students interviewed having greater interest in and knowledge about mental illnesses and schizophrenia. The use of snowball sampling ${ }^{20}$ could have further led to similarities between participants within ethnicities and potential bias. It is important to note this study interviewed 20 students with a maximum of five from each ethnicity and therefore the views portrayed cannot be taken to reflect the views of entire communities. Finally, owing to some participants' lack of knowledge about schizophrenia, interviews were as short as $15 \mathrm{~min}$.

\section{Research and policy recommendations}

Further research involving participants with a range of educational levels would provide a better understanding of the level of public knowledge. This study, alongside previous research, does however demonstrate there are low levels of understanding. Campaigns educating the public are therefore crucial to improve knowledge which may aid earlier consultation. Educating the public and schools on the signs and symptoms of schizophrenia has previously proven successful in reducing duration of untreated psychosis when employed in Norway and Denmark. ${ }^{40}$ Education on availability of treatment and benefits of early intervention may further aid this.

Despite recent anti-stigma campaigns ${ }^{18}$ significant stigma was apparent. Further campaigns therefore require careful consideration, with research required into which strategies effectively reduce stigma associated with schizophrenia to aid successful future campaigns. Education and anti-stigma campaigns targeted to ethnicities may prove of use. Campaigns addressing the 'taboo' of schizophrenia in minority ethnic groups could allow earlier help-seeking and greater community support. Education within the Indian and Pakistani community on schizophrenia as a real illness separate to possession by a spirit could further increase understanding and support.

\section{CONCLUSION}

This study demonstrates the confusion and negative connotations surrounding schizophrenia in a selection of university students in Birmingham, UK. Public health campaigns to better educate them and the wider public about schizophrenia, including its symptoms, to aid early identification and intervention and improve outcomes is necessary. Further research is also required to design and develop effective strategies to tackle the stigma surrounding schizophrenia.

Acknowledgements I would like to thank all the participants for their contribution to the study and Dr Lindenmeyer for her guidance during analysis.

Contributors CCa designed the study, wrote the study protocol, obtained ethical approval, undertook recruitment, carried out interviews, analysed the data and drafted the manuscript. SG provided expert supervision contributing to the study design, protocol and analysis through acting as the additional analyst. SG and CCo read through and edited the transcript. CCa also read through and edited the paper.

Funding This research was funded by the BMedSc Population Sciences and Humanities Programme at the University of Birmingham. SG and CCo are part funded by the National Institute for Health Research (NIHR) Collaboration for Leadership in Applied Health Research and Care West Midlands.
Disclaimer The views expressed in this article are those of the author(s) and not necessarily those of the NHS, the NIHR, or the Department of Health and Social Care.

\section{Competing interests None declared.}

Patient consent for publication Not required.

Ethics approval Ethical approval was granted by the BMedSc Population Sciences and Humanities Internal Ethics Review Committee at the University of Birmingham.

Provenance and peer review Not commissioned; externally peer reviewed.

Data sharing statement No additional data available.

Open access This is an open access article distributed in accordance with the Creative Commons Attribution Non Commercial (CC BY-NC 4.0) license, which permits others to distribute, remix, adapt, build upon this work non-commercially, and license their derivative works on different terms, provided the original work is properly cited, appropriate credit is given, any changes made indicated, and the use is non-commercial. See: http://creativecommons.org/licenses/by-nc/4.0/.

\section{REFERENCES}

1. National Institue for Clinical Excellence. Psychosis and schizophrenia in adults: prevention and management; Clinical Guidelines [CG178]. 2014 https://www.nice.org.uk/guidance/cg178/chapter/Keypriorities-for-implementation (Accessed Apr 2016).

2. McGlashan TH, Johannessen JO. Early detection and intervention with schizophrenia: rationale. Schizophr Bull 1996;22:201-22.

3. Perkins DO, Gu H, Boteva K, et al. Relationship between duration of untreated psychosis and outcome in first-episode schizophrenia: a critical review and meta-analysis. Am J Psychiatry 2005;162:1785-804.

4. Penttilä $M$, Jääskeläinen $E$, Hirvonen $N$, et al. Duration of untreated psychosis as predictor of long-term outcome in schizophrenia: systematic review and meta-analysis. Br J Psychiatry 2014;205:88-94.

5. Birchwood M, Connor C, Lester $\mathrm{H}$, et al. Reducing duration of untreated psychosis: care pathways to early intervention in psychosis services. Br J Psychiatry 2013;203:58-64.

6. Marshall M, Lewis S, Lockwood A, et al. Association between duration of untreated psychosis and outcome in cohorts of first-episode patients: a systematic review. Arch Gen Psychiatry 2005;62:975-83.

7. Jorm AF, Korten AE, Jacomb PA, et al. "Mental health literacy": a survey of the public's ability to recognise mental disorders and their beliefs about the effectiveness of treatment. Med J Aust 1997;166:182-6.

8. Connor C, Birchwood M, Freemantle N, et al. Don't turn your back on the symptoms of psychosis: the results of a proof-of-principle, quasiexperimental intervention to reduce duration of untreated psychosis. BMC Psychiatry 2016;16:127.

9. Baier M. Insight in schizophrenia: a review. Curr Psychiatry Rep 2010;12:356-61.

10. World Health Organization. Report of the international pilot study of schizophrenia. Geneva: World Health Organization, 1973.

11. Angermeyer MC, Dietrich S. Public beliefs about and attitudes towards people with mental illness: a review of population studies. Acta Psychiatr Scand 2006;113:163-79.

12. Furnham A, Cook R, Martin N, et al. Mental health literacy among university students. J Public Ment Health 2011;10:198-210.

13. Royal College of Psychiatrists. Mental health of students in higher education. 2011 https://www.rcpsych.ac.uk/files/pdfversion/cr166. pdf (Accessed Oct 2018).

14. Scior K, Potts HW, Furnham AF. Awareness of schizophrenia and intellectual disability and stigma across ethnic groups in the UK. Psychiatry Res 2013;208:125-30.

15. Erritty $P$, Wydell TN. Are lay people good at recognising the symptoms of schizophrenia? PLoS One 2013;8:e52913.

16. Stone L, Finlay WM. A comparison of African-Caribbean and White European young adults' conceptions of schizophrenia symptoms and the diagnostic label. Int J Soc Psychiatry 2008;54:242-61.

17. Cinnirella M, Loewenthal KM. Religious and ethnic group influences on beliefs about mental illness: a qualitative interview study. $\mathrm{Br} J$ Med Psychol 1999;72:505-24.

18. Henderson $\mathrm{C}$, Thornicroft G. Evaluation of the time to change programme in England 2008-2011. Br J Psychiatry Suppl 2013;55:s45-8.

19. Pote HL, Orrell MW. Perceptions of schizophrenia in multi-cultural Britain. Ethn Health 2002;7:7-20. 
20. Robinson OC. Sampling in interview-based qualitative research: a theoretical and practical guide. Qual Res Psychol 2014;11:25-41.

21. Office for National Statistics. Ethnicity and National Identity in England and Wales - Office for National Statistics. 2012 https://www. ons.gov.uk/peoplepopulationandcommunity/culturalidentity/ethnicity/ articles/ethnicityandnationalidentityinenglandandwales/2012-12-11 (Accessed Apr 2017).

22. Thornton L, Batterham PJ, Fassnacht DB, et al. Recruiting for health, medical or psychosocial research using Facebook: Systematic review. Internet Interv 2016;4:72-81.

23. Morse JM. Determining Sample Size. Qual Health Res 2000;10:3-5.

24. Braun V, Clarke V. Using thematic analysis in psychology. Qual Res Psychol 2006;3:77-101.

25. Denzin NK, Lincoln YS. The SAGE Handbook of qualitative research. Fourth edn. Los Angeles: Sage Publications, 2011.

26. Patton $M Q$. Enhancing the quality and credibility of qualitative analysis. Health Serv Res 1999;34:1189-208.

27. Maxwell JA. Using numbers in qualitative research. Qualitative Inquiry 2010;16:475-82.

28. Islam E-ZA. Arabs and the intelligent world of the jinn: Syracuse University Press, 2009.

29. Larson MK, Walker EF, Compton MT. Early signs, diagnosis and therapeutics of the prodromal phase of schizophrenia and related psychotic disorders. Expert Rev Neurother 2010;10:1347-59.

30. Gourzis P, Katrivanou A, Beratis S. Symptomatology of the initial prodromal phase in schizophrenia. Schizophr Bull 2002;28:415-29.
31. Luty J, Fekadu D, Dhandayudham A. Understanding of the term "schizophrenia"by the British public. World Psychiatry 2006;5:177-8.

32. Walsh E, Buchanan A, Fahy T. Violence and schizophrenia: examining the evidence. Br J Psychiatry 2002;180:490-5.

33. Gorczynski P, Sims-schouten W, Hill D, et al. Examining mental health literacy, help seeking behaviours, and mental health outcomes in UK university students. J of Ment Health Training, Education and Practice 2017;12:111-20.

34. Connor C, Greenfield S, Lester $\mathrm{H}$, et al. Seeking help for first-episode psychosis: a family narrative. Early Interv Psychiatry 2016;10:334-45.

35. Harrison G, Hopper K, Craig T, et al. Recovery from psychotic illness: a 15- and 25-year international follow-up study. Br J Psychiatry 2001;178:506-17.

36. Goffman E. Stigma: notes on the management of spoiled identity. New York: Prentice Hall, 1963.

37. U.S Department of Health and Human Services. Mental Health: Culture, Race, and Ethnicity - A Supplement to Mental Health: A Report of the Surgeon General. Department of health and human services, substance abuse and mental health services administration, center for mental health services. Rockville, MD.

38. McCabe R, Priebe S. Explanatory models of illness in schizophrenia: comparison of four ethnic groups. Br J Psychiatry 2004;185:25-30.

39. Smart A, Harrison $E$. The under-representation of minority ethnic groups in UK medical research. Ethn Health 2017;22:65-82.

40. Joa I, Johannessen JO, Auestad B, et al. The key to reducing duration of untreated first psychosis: information campaigns. Schizophr Bull 2008;34:466-72. 